\title{
FBXW7 inhibits invasion, migration and angiogenesis in ovarian cancer cells by suppressing VEGF expression through inactivation of $\beta$-catenin signaling
}

\author{
LIPING ZHONG, YUEFEN PAN and JUNJUN SHEN
}

\author{
Department of Medical Oncology, Huzhou Central Hospital, \\ Affiliated Central Hospital Huzhou University, Huzhou, Zhejiang 313000, P.R. China
}

Received November 24, 2020; Accepted February 26, 2021

DOI: $10.3892 /$ etm.2021.9945

\begin{abstract}
F-box and WD repeat domain containing 7 (FBXW7) is a tumor suppressor gene frequently inactivated in several human malignancies. The present study aimed to investigate the role of FBXW7 in the invasion, migration and angiogenesis of ovarian cancer (OC) cells, and to identify its potential molecular mechanisms. First, the expression levels of FBXW7 and vascular endothelial growth factor (VEGF) were detected in several human OC cell lines using western blotting. Subsequently, FBXW7 was overexpressed to determine VEGF expression in SKOV3 cells. Transwell, wound healing and tube formation assays were performed following transfection with FBXW7 and VEGF overexpression plasmids to assess invasion, migration and angiogenesis in SKOV3 cells, respectively. Western blot analysis was performed to detect the expression levels of epithelial-to-mesenchymal transition and angiogenesis-associated proteins. In addition, the expression levels of $\beta$-catenin and c-Myc were assessed, and lithium chloride $(\mathrm{LiCl})$, an agonist of $\beta$-catenin signaling, was used to elucidate the molecular mechanisms by which FBXW7 mediates its antitumor activity in OC. The results demonstrated that FBXW7 expression was markedly downregulated, whilst VEGF expression was markedly upregulated in OC cell lines compared with that in normal ovarian cells. Overexpression of FBXW7 significantly decreased VEGF expression in SKOV3 cells. Notably, overexpression of VEGF reversed the inhibitory effects of FBXW7 overexpression on the invasion, migration and angiogenesis of OC cells, accompanied by upregulated expression levels of N-cadherin, slug, CD31, VEGF receptor 1 (VEGFR1) and VEGFR2, and downregulated expression levels of E-cadherin. Furthermore,
\end{abstract}

Correspondence to: Dr Junjun Shen, Department of Medical Oncology, Huzhou Central Hospital, Affiliated Central Hospital Huzhou University, 1558 Sanhuan North Road, Wuxing, Huzhou, Zhejiang 313000, P.R. China

E-mail: shengjunjsjj@163.com

Key words: ovarian cancer, angiogenesis, F-box and WD repeat domain containing 7 , vascular endothelial growth factor, $\beta$-catenin overexpression of FBXW7 markedly suppressed $\beta$-catenin and c-Myc expression, whereas the decreased expression levels of VEGF, VEGFR1 and VEGFR2 following overexpression of FBXW7 were increased after treatment of SKOV3 cells with $\mathrm{LiCl}$. Overall, the results of the present study suggested that FBXW7 inhibited invasion, migration and angiogenesis of OC cells by suppressing VEGF expression through inactivation of $\beta$-catenin signaling. Thus, FBXW7 may be used as a novel therapeutic target for the treatment of OC.

\section{Introduction}

Ovarian cancer (OC) is one of the deadliest gynecological malignancies, with high incidence and mortality rates worldwide $(1,2)$. There were $\sim 239,000$ new cases and 152,000 deaths worldwide annually with regard to this disease in 2017 (3). Despite advancements in treatment options, the 5-year survival rate of patients with $\mathrm{OC}$ remains $<35 \%$ globally due to distant metastasis and recurrence $(4,5)$. Thus, it is urgent to obtain an improved understanding regarding the pathogenesis underlying OC to identify and develop more effective therapeutic targets for the treatment of this disease.

F-box and WD repeat domain containing 7 (FBXW7), a member of the F-box protein family, is an evolutionarily conserved F-box protein, containing two vital functional domains (F-box and WD), which are indispensable for its function (6). FBXW7 is a critical tumor suppressor gene, where mutations within this gene have been implicated in different types of human cancer $(7,8)$. For instance, FBXW7 can target salt inducible kinase 2 for degradation, leading to the disruption of target of rapamycin 2-AKT signaling to inhibit pancreatic cancer cell proliferation and cell cycle progression (9). It has also been reported that FBXW7 suppresses oral squamous cell carcinoma proliferation and invasion regulated by miR-27a through the PI3K/AKT signaling pathway (10). Increasing evidence has suggested that FBXW7 serves as a key regulator in the proliferation, invasion, migration and apoptosis of human cancer cells through the degradation of oncoproteins, including c-Myc, in a proteasome-dependent manner $(11,12)$. Previous studies have demonstrated that FBXW7 is deleted or methylated in epithelial OC, and its expression is negatively associated with the malignant potential of ovarian tumors $(13,14)$. FBXW7 
has been reported to act as a positive regulator of angiogenesis in the endothelium of the growing vasculature (15). Increasing evidence has suggested that angiogenesis serves a crucial role in the invasion, migration and metastasis of $\mathrm{OC}$, with a positive association between the rate and extent of angiogenesis and an unfavorable prognosis in patients with OC $(16,17)$. Vascular endothelial growth factor (VEGF), a homodimeric glycoprotein, is the key mediator of angiogenesis, which binds two important VEGF receptors, VEGFR1 and VEGFR2 $(18,19)$. However, the effects of FBXW7 on the angiogenesis of OC, and whether FBXW7 functions by regulating VEGF expression, remain unclear.

The present study aimed to investigate VEGF expression following overexpression of FBXW7 in OC cells. In addition, the role of FBXW7 on the invasion, migration and angiogenesis of OC cells, and its potential regulatory effects on VEGF expression were assessed.

\section{Materials and methods}

Cell culture. The normal human ovarian epithelial cell line (IOSE-80) and several OC cell lines (ES-2, SKOV3, PA1 and OVCAR3) were purchased from the American Type Culture Collection and maintained in DMEM (Invitrogen; Thermo Fisher Scientific, Inc.) supplemented with 10\% FBS (HyClone; Cytiva) at $37^{\circ} \mathrm{C}$ with $5 \% \mathrm{CO}_{2}$. Cells were treated with the $\beta$-catenin activator, lithium chloride $(\mathrm{LiCl} ; 20 \mathrm{mM}$; Sigma-Aldrich; Merck KGaA) for $3 \mathrm{~h}$ at $37^{\circ} \mathrm{C}$.

Human umbilical vein endothelial cells (HUVECs) were provided The Cell Bank of Type Culture Collection of the Chinese Academy of Sciences and cultured in a mixture containing RPMI-1640 medium (HyClone; Cytiva) and 10\% FBS. The cells were incubated at $37^{\circ} \mathrm{C}$ in a humidified atmosphere which was maintained at $5 \% \mathrm{CO}_{2}$.

Cell transfection. SKOV3 cells were seeded into 6-well plates at a density of $2 \times 10^{5}$ cells/well and cultured at $37^{\circ} \mathrm{C}$ until they reached $80 \%$ confluence. pcDNA 3.1 containing FBXW7 (Ov-FBXW7) or VEGF (pc-VEGF), and the corresponding empty vectors used as negative controls (Ov-NC and pc-NC, respectively) were synthesized by Shanghai GenePharma Co., Ltd. SKOV3 cells were transfected with the respective plasmids (50 nM) using Lipofectamine ${ }^{\circledR} 2000$ reagent (Invitrogen; Thermo Fisher Scientific, Inc.) at $37^{\circ} \mathrm{C}$ for $24 \mathrm{~h}$, according to the manufacturer's instructions. Cells were collected and the transfection efficiency was assessed via reverse transcription-quantitative (RT-q) PCR and western blot analyses $24 \mathrm{~h}$ post-transfection.

Invasion assay. Cell invasion was assessed using Transwell assay (pore size, $8.0 \mu \mathrm{m}$; Corning Inc.) precoated with Matrigel $\left(6.25 \mathrm{mg} / \mathrm{l}\right.$; BD Biosciences) overnight at $4^{\circ} \mathrm{C}$. A total of $2 \times 10^{5}$ transfected SKOV3 cells were resuspended in $200 \mu 1$ serum-free DMEM and plated in the upper chambers of Transwell plates, whilst $600 \mu 1$ DMEM supplemented with $10 \%$ FBS was used as the chemoattractant and plated in the lower chambers. Following incubation for $24 \mathrm{~h}$ at $37^{\circ} \mathrm{C}$, the invasive cells were fixed with $4 \%$ formaldehyde for $30 \mathrm{~min}$ at room temperature and subsequently stained with $0.1 \%$ crystal violet for $30 \mathrm{~min}$ at room temperature. Stained cells were counted in five randomly selected fields using an inverted light microscope (Olympus Corporation; magnification, x100) and the results were analyzed using ImageJ software (version 1.52r; National Institutes of Health).

Wound healing assay. Cell migration was assessed using wound healing assays. Briefly, SKOV3 cells were seeded into 6 -well plates at a density of $4 \times 10^{5}$ cells/well and cultured in DMEM supplemented with $10 \%$ FBS until they reached $80 \%$ confluence. The cell monolayers were scratched using sterile $200-\mu 1$ pipette tips. Cells were washed with PBS to elute the debris, and the medium was replaced with serum-free DMEM. Following incubation for $24 \mathrm{~h}$ at $37^{\circ} \mathrm{C}$, the average distance of cells migrated into the wound surface was observed under an inverted light microscope (Olympus Corporation; magnification, x100). Quantitative analysis of the wound healing area was performed using ImageJ software (version 1.52r; National Institutes of Health).

Tube formation assay. A total of $1.5 \times 10^{4}$ HUVECs were seeded into 96-well plates precoated with Matrigel $(10 \mathrm{mg} / \mathrm{ml}$; BD Biosciences) at $4^{\circ} \mathrm{C}$ overnight and incubated for $6 \mathrm{~h}$ at $37^{\circ} \mathrm{C}$ in a $5 \% \mathrm{CO}_{2}$ incubator with the supernatants from SKOV3 cells transfected with Ov-FBXW7 or/and pc-VEGF. Tube formation was observed under an inverted light microscope (Olympus Corporation). The number of loops formed was counted in five randomly selected fields and analyzed using ImageJ software (version 1.52r; National Institutes of Health). A connection between two cells was counted as one capillary tube formation.

$R T-q P C R$. Total RNA was extracted from SKOV3 cells using TRIzol ${ }^{\circledR}$ reagent (Thermo Fisher Scientific, Inc.). Total RNA was reverse transcribed into cDNA using the First Strand cDNA synthesis kit (Thermo Fisher Scientific, Inc.), according to the manufacturer's protocol. qPCR was subsequently performed with $2 \mu \mathrm{g}$ cDNA using the SYBR Premix Ex Taq (Takara Bio, Inc.) and ABI 7500 equipment (Applied Biosystems; Thermo Fisher Scientific, Inc.). The following thermocycling conditions were used: Initial denaturation at $95^{\circ} \mathrm{C}$ for $10 \mathrm{~min}$; followed by 40 cycles of denaturation at $95^{\circ} \mathrm{C}$ for $15 \mathrm{sec}$ and annealing at $60^{\circ} \mathrm{C}$ for $1 \mathrm{~min}$; and a final extension of $10 \mathrm{~min}$ at $72^{\circ} \mathrm{C}$. The sequences of the gene-specific primers used in the present study were as follows FBXW7 forward, 5'-CACAGG CCTTCAAGAGTGGC-3' and reverse, 5'-TTGCATCATATG CTTCACTTGTGT-3'; VEGF forward, 5'-GGGCAGAATCAT CACGAAGT-3' and reverse, 5'-AAATGCTTTCTCCGCTC TGA-3'; CD31 forward, 5'-TGCAGTGGTTATCATCGGA GTG-3' and reverse, 5'-CGTTGTTGGAGTTCAGAAGTG-3'; VEGFR1 forward, 5'-TGCCTCAGAAGAGCTGAAAAC-3' and reverse, 5'-CACAGACTCCCTGCTTTTGCT-3'; VEGFR2 forward, 5'-GCACATTGGTGGTGGCTGAC-3' and reverse, 5'-CTCTCCTTCGGCTGGCATCT-3' and GAPDH forward, 5'-ACAACTTTGGTATCGTGGAAGG-3' and reverse, 5'-GCCATCACGCCACAGTTTC-3'. Relative expression levels were calculated using the $2^{-\Delta \Delta C q}$ method and normalized to the internal reference gene GAPDH (20).

Western blotting. Total protein was extracted from SKOV3 cells using RIPA lysis buffer (Beyotime Institute of Biotechnology). Total protein was quantified using the bicinchoninic acid kit (Beyotime Institute of Biotechnology) and 

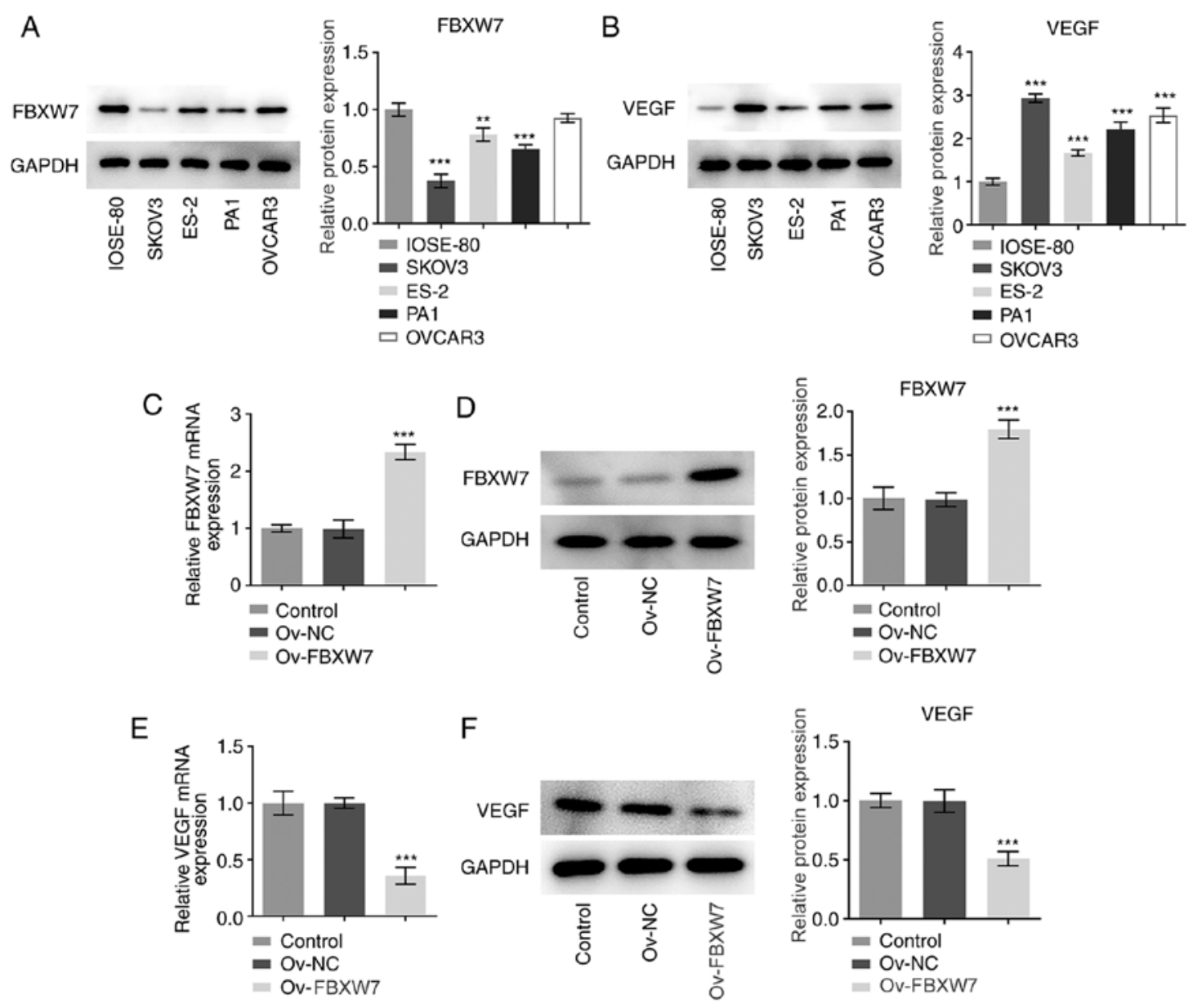

Figure 1. Overexpression of FBXW7 significantly decreases VEGF expression in SKOV3 cells. Protein expression levels of (A) FBXW7 and (B) VEGF in a normal human ovarian epithelial cell line (IOSE-80) and several OC cell lines (ES-2, SKOV3, PA1 and OVCAR3) were detected via western blot analysis. ${ }^{* *} \mathrm{P}<0.01$ and ${ }^{* * * *} \mathrm{P}<0.001$ vs. IOSE-80. (C) RT-qPCR and (D) western blot analyses were performed to detect FBXW7 expression following transfection with Ov-FBXW7. (E) RT-qPCR and (F) western blot analyses were performed to detect VEGF expression following overexpression of FBXW7. ${ }^{* * * *} \mathrm{P}<0.001$ vs. Ov-NC. FBXW7, F-box and WD repeat domain containing 7; VEGF, vascular endothelial growth factor; RT-qPCR, reverse transcription-quantitative PCR; Ov, overexpression; NC, negative control.

$4 \mu \mathrm{g}$ protein/lane was separated by $10 \%$ SDS-PAGE. The separated proteins were subsequently transferred onto polyvinylidene fluoride membranes (EMD Millipore) and blocked with $5 \%$ skimmed milk for $1.5 \mathrm{~h}$ at room temperature. The membranes were incubated with corresponding primary antibodies at $4^{\circ} \mathrm{C}$ overnight. Following the primary antibody incubation, membranes were incubated with a goat anti-rabbit horseradish peroxidase (HRP)-conjugated secondary antibody (1:3,000; cat. no. 7074S; Cell Signaling Technology, Inc.) or horse anti-mouse HRP-conjugated secondary antibody (1:3,000; cat. no. 7076S; Cell Signaling Technology) for $1.5 \mathrm{~h}$ at room temperature. The immunoreactive protein bands on the membranes were visualized using an enhanced chemiluminescence assay (EMD Millipore). The relative intensity of target bands were semi-quantified using ImageJ software (version 1.52r; National Institutes of Health) and normalized by the intensity of GAPDH. Anti-FBXW7 (cat. no. ab109617; 1:1,000) antibody was provided by Abcam. Anti-VEGF (cat. no. 2463S; 1:1,000), anti-CD31 (cat. no. 3528S; 1:1,000), anti-VEGFR1 (cat. no. 64094S; 1:1,000), VEGFR2 (cat. no. $9698 \mathrm{~S} ; 1: 1,000)$, anti-E-cadherin (E-cad; cat. no. 3195T; 1:1,000), anti-N-cadherin (N-cad; cat. no. 13116T; 1:1,000), anti-Slug (cat. no. 9585T; 1:1,000) and anti-GAPDH (cat. no. 5174T; 1:1,000) antibodies were all purchased from Cell Signaling Technology, Inc.

Statistical analysis. All experiments were repeated three times independently. Statistical analysis was performed using GraphPad Prism 8.0 (GraphPad Software, Inc.). Data are presented as the mean $\pm \mathrm{SD}$. An unpaired Student's t-test was used to compare differences between two groups, while one-way ANOVA followed by Tukey's post hoc test was used to compare differences among multiple groups. $\mathrm{P}<0.05$ was considered to indicate a statistically significant difference.

\section{Results}

Overexpression of FBXW7 significantly downregulates $V E G F$ expression in $O C$ cells. Firstly, the expression levels of FBXW7 and VEGF in a normal human ovarian epithelial cell line (IOSE-80) and several OC cell lines (ES-2, SKOV3, PA1 and OVCAR3) were detected via western blot analysis. As shown in Fig. 1A and B, FBXW7 expression was markedly downregulated, while VEGF expression was significantly upregulated in OC cell lines compared with in IOSE-80 cells, especially in SKOV3 cells, which were therefore used 
A

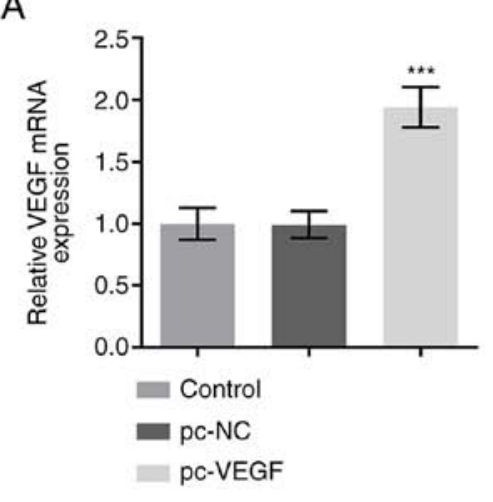

B

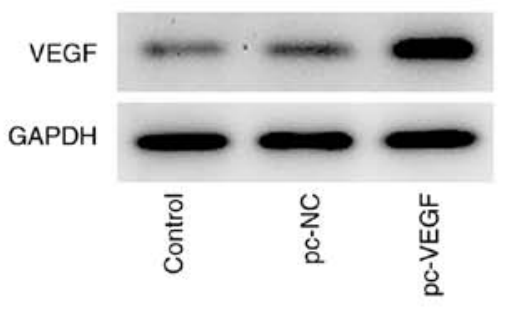

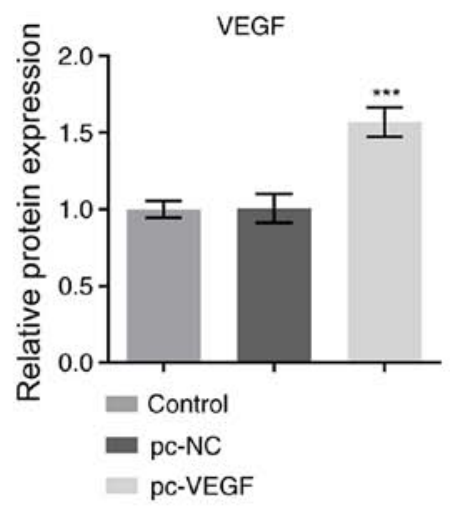

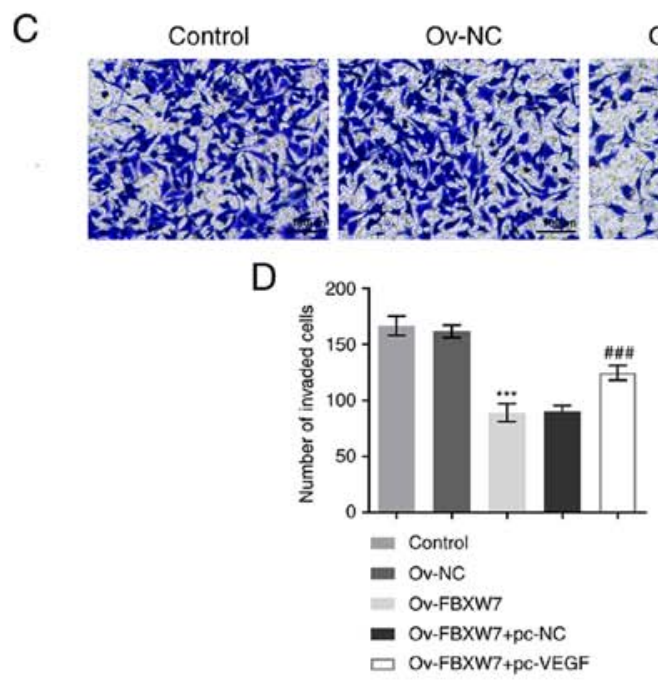

F
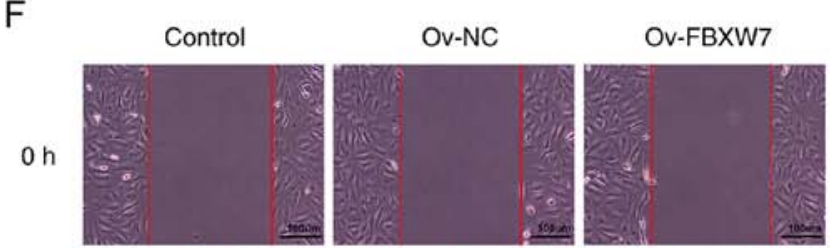

E

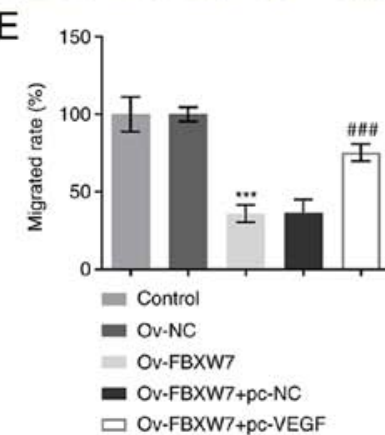

$24 \mathrm{~h}$
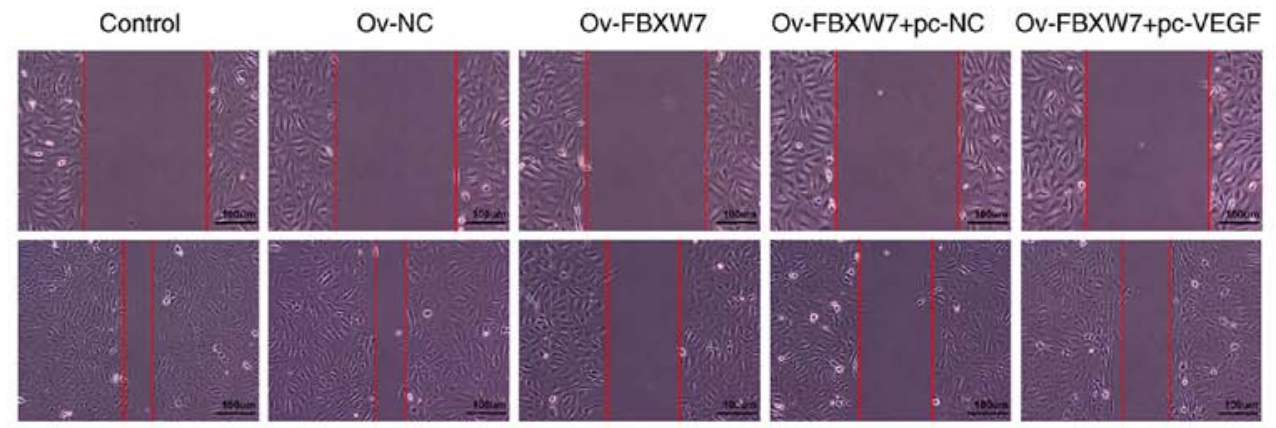

Figure 2. Overexpression of VEGF attenuates the inhibitory effects of FBXW7 overexpression on the invasion and migration of SKOV3 cells. (A) Reverse transcription-quantitative PCR and (B) western blot analyses were performed to detect VEGF expression following transfection with the VEGF plasmid. (C and D) Transwell assay was performed to assess the invasive ability of SKOV3 cells. (E and F) Wound healing assay was performed to assess the migratory ability of SKOV3 cells. Scale bar, $100 \mu \mathrm{m}$. ${ }^{* * *} \mathrm{P}<0.001$ vs. pc-NC or Ov-NC; ${ }^{\# \#} \mathrm{P}<0.001$ vs. Ov-FBXW7+pc-NC. VEGF, vascular endothelial growth factor; FBXW7, F-box and WD repeat domain containing 7; Ov, overexpression; NC, negative control.

in subsequent experiments. Subsequently, FBXW7 was overexpressed in SKOV3 cells. As presented in Fig. 1C and D, FBXW7 expression was significantly upregulated at both the transcriptional and protein levels following transfection with Ov-FBXW7 compared with those after transfection with Ov-NC. Notably, overexpression of FBXW7 significantly decreased VEGF mRNA and protein expression compared with the vector control group (Fig. 1E and F). Overall, these results suggested that overexpression of FBXW7 inhibited VEGF expression in OC cells.

Overexpression of VEGF restores the inhibitory effects of FBXW7 overexpression on the invasion, migration and epithelial-to-mesenchymal transition (EMT) of OC cells. To determine whether FBXW7 regulates VEGF expression in OC, VEGF was overexpressed following transfection with a plasmid containing VEGF. As presented in Fig. 2A and B, VEGF mRNA and protein expression was significantly increased in the pc-VEGF group compared with in the pc-NC group.

Transwell and wound healing assays were performed to assess the invasive and migratory abilities of SKOV3 cells, respectively. As presented in Fig. 2C-F, overexpression of FBXW7 significantly inhibited the invasive and migratory abilities of SKOV3 cells compared with the Ov-NC group. Conversely, co-transfection with VEGF and FBXW7 overexpression plasmids significantly promoted the 

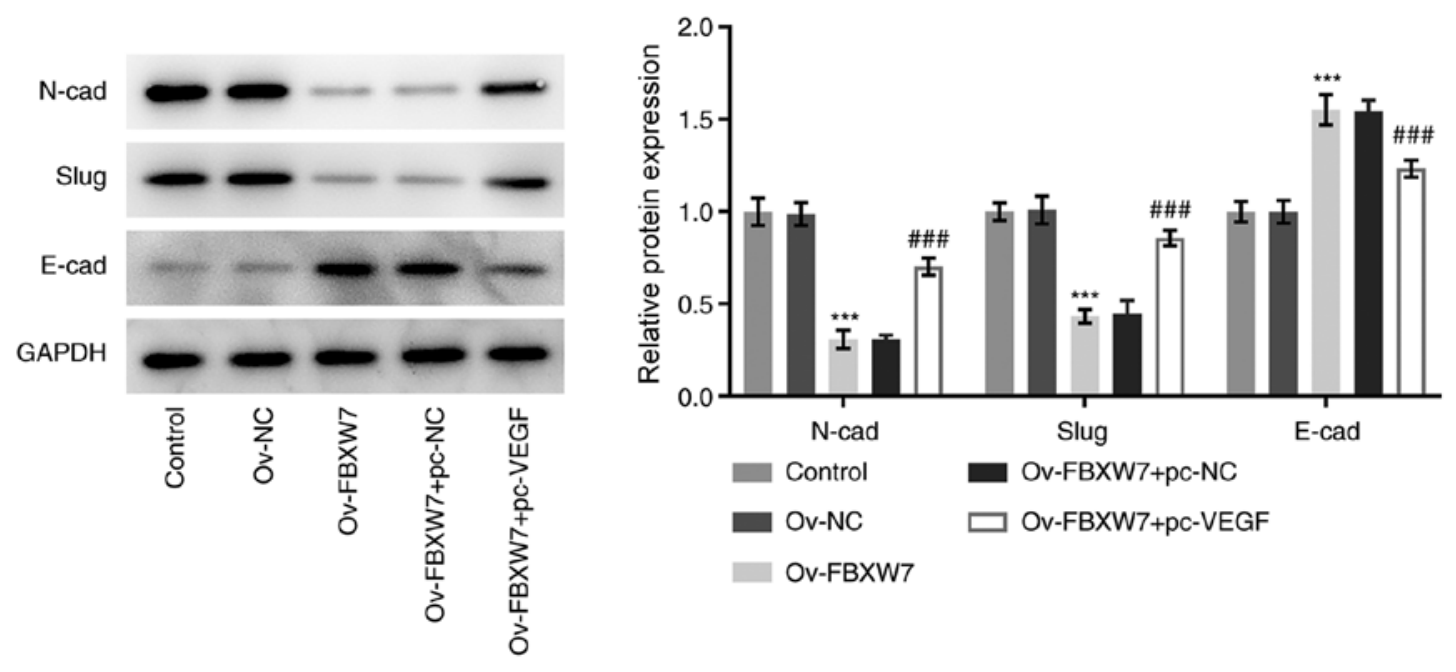

Figure 3. Overexpression of VEGF reverses the inhibitory effects of FBXW7 overexpression on the EMT process of SKOV3 cells. Western blot analysis was performed to detect the expression levels of EMT-associated proteins, including N-cad, slug and E-cad. ${ }^{* * *} \mathrm{P}<0.001$ vs. Ov-NC; ${ }^{\# \# \# ~ P<0.001 ~ v s . ~ O v-F B X W 7+p c-N C . ~}$ EMT, epithelial-to-mesenchymal transition; FBXW7, F-box and WD repeat domain containing 7; VEGF, vascular endothelial growth factor; Ov, overexpression; NC, negative control; N/E-cad, N/E-cadherin.

invasive and migratory abilities of SKOV3 cells compared with cells transfected with the FBXW7 overexpression plasmid alone (Fig. 2C-F). Additionally, overexpression of FBXW7 significantly decreased the expression levels of $\mathrm{N}$-cadherin and slug, while significantly increasing E-cadherin expression, and these effects were reversed following co-transfection with the VEGF overexpression plasmid (Fig. 3). Collectively, these results indicated that overexpression of FBXW7 inhibited the invasion, migration and EMT process of OC cells by suppressing VEGF expression.

Overexpression of VEGF partially counteracts the impact of FBXW7 overexpression on the angiogenesis of OC cells. To investigate the effect of FBXW7 on the angiogenesis of OC cells, a tube formation assay was performed. As presented in Fig. 4A and B, there was no significant difference in the number of tubes formed between the control and the Ov-NC groups. However, a significantly decreased number of tubes was observed in the Ov-FBXW7 group compared with in the Ov-NC group (Fig. 4A and B). Notably, overexpression of VEGF enhanced the number of tubes formed compared with the Ov-FBXW7+pc-NC group (Fig. 4A and B). RT-qPCR and western blot analyses were performed to detect the expression levels of proteins associated with angiogenesis. The results demonstrated that overexpression of FBXW7 downregulated the expression levels of CD31, VEGFR1 and VEGFR, whereas co-transfection with FBXW7 and VEGF plasmids significantly increased their expression levels compared with the Ov-FBXW7+pc-NC group (Fig. 4C and D), which is consistent with the results of the tube formation assay. Overall, these results suggested that overexpression of FBXW7 suppressed the angiogenesis of OC cells by suppressing VEGF expression.

FBXW7 inhibits VEGF expression through inactivation of $\beta$-catenin signaling. To further elucidate the potential molecular mechanism by which FBXW7 mediates its antitumor effects in OC, western blot analysis was performed to detect the expression levels of key proteins in $\beta$-catenin signaling. As presented in Fig. 5A, overexpression of FBXW7 significantly decreased the expression levels of $\beta$-catenin and c-Myc compared with the empty vector group. Furthermore, $\mathrm{LiCl}$, an agonist of $\beta$-catenin signaling, was used to treat SKOV3 cells transfected with the FBXW7 plasmid, and the expression levels of angiogenesis-associated proteins were determined. As presented in Fig. 5B, treatment with $\mathrm{LiCl}$ significantly abrogated the inhibitory effects of FBXW7 overexpression on the expression levels of VEGF, VEGFR1 and VEGFR2. Collectively, these results suggested that FBXW7 may inhibit VEGF expression through inactivation of $\beta$-catenin signaling in SKOV3 cells.

\section{Discussion}

$\mathrm{OC}$ is one of the most common and lethal types of cancer in women, and has been a significant public health burden worldwide (21). Thus, it is of great importance to further understand the molecular mechanisms of OC tumorigenesis and progression to identify and develop effective therapeutic strategies. The results of the present study demonstrated that FBXW7 efficiently inhibited SKOV3 cell invasion and migration, as well as tube formation of HUVECs. Mechanistically, FBXW7 suppressed VEGF expression by inactivating $\beta$-catenin signaling.

Invasion and migration are two hallmarks of the malignant biological behavior of $\mathrm{OC}$, and interdiction of these progresses is a critical factor to improve biomedical treatment worldwide $(22,23)$. EMT, a process in which stationary epithelial cells attain a highly active mesenchymal phenotype, is an essential and important step in tumor cell invasion, migration and relocalization (24-26). Downregulated expression levels of E-cadherin (a crucial epithelial marker) in epithelial cells, along with upregulated expression levels of mesenchymal proteins, including $\mathrm{N}$-cadherin and slug, are common hallmarks of EMT $(27,28)$. Angiogenesis refers to the generation of new blood vessels by the sprouting of endothelial cells from preexisting ones (29). It has been reported that tumors depend on the constant growth of new blood vessels, whereby interruption of the blood supply may eliminate the cancer (30). 
A

Control

Ov-NC

Ov-FBXW7

Ov-FBXW7+pc-NC

Ov-FBXW7+pc-VEGF
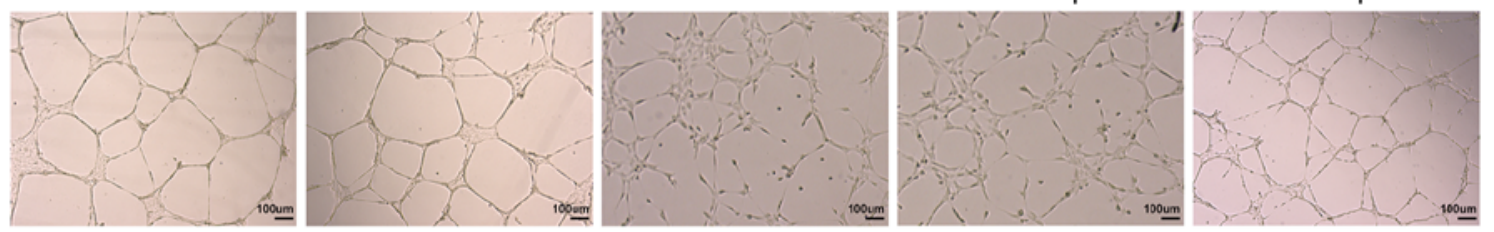

B

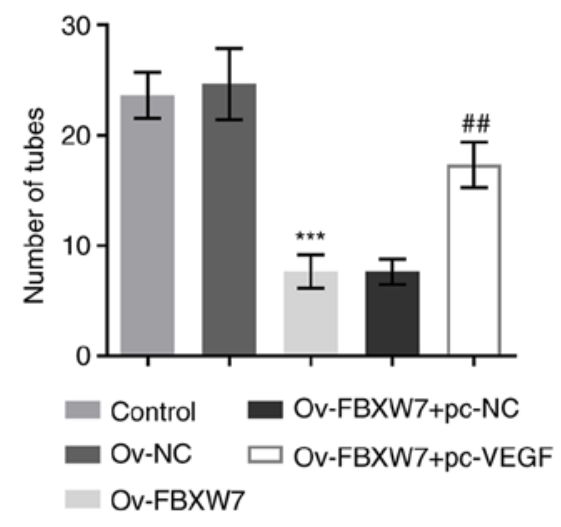

C

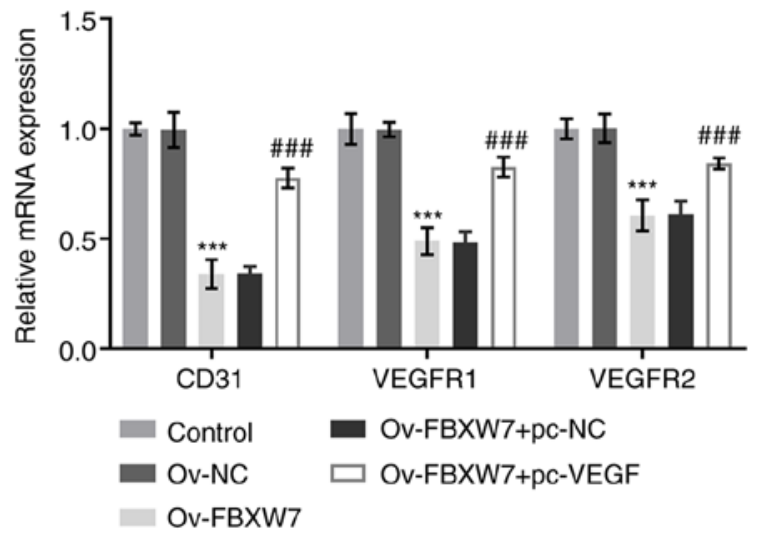

D
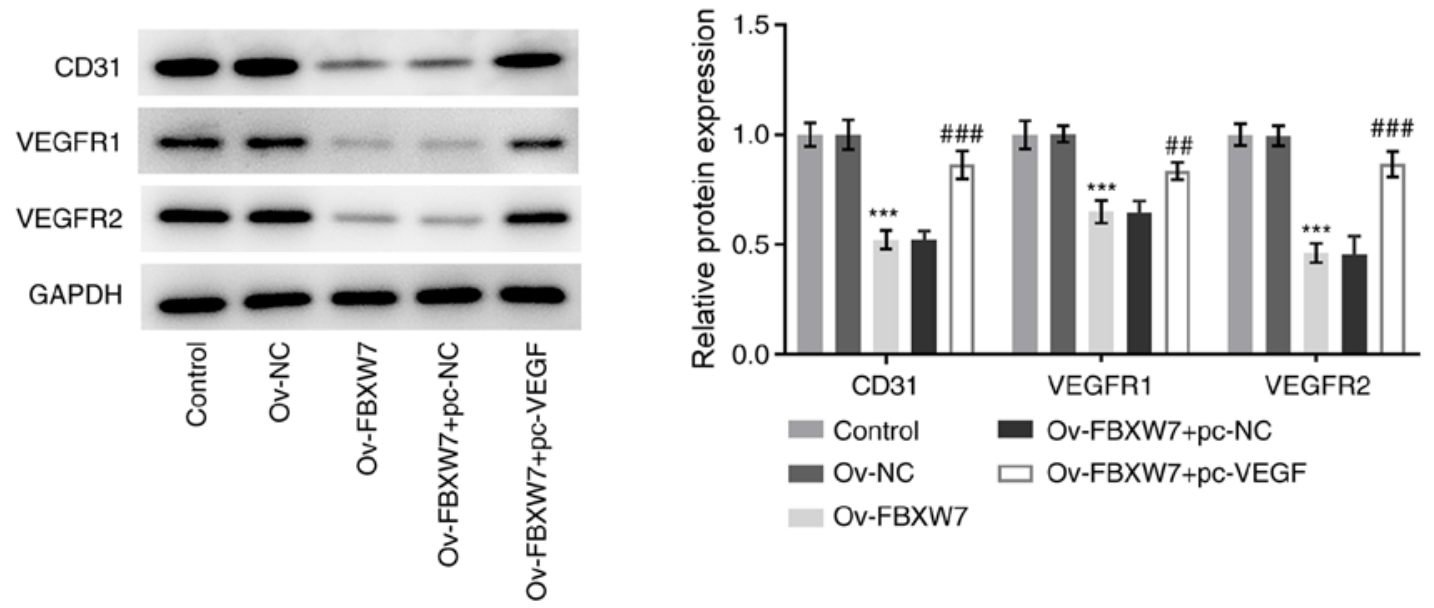

Figure 4. Overexpression of VEGF abrogates the effects of FBXW7 overexpression on the angiogenesis of SKOV3 cells. (A and B) Tube formation assay was performed to assess angiogenesis. (C) Reverse transcription-quantitative PCR and (D) western blot analyses were performed to detect the expression levels of angiogenesis-associated proteins. ${ }^{* * *} \mathrm{P}<0.001$ vs. Ov-NC; ${ }^{\# \#} \mathrm{P}<0.01,{ }^{\# \# \#} \mathrm{P}<0.001$ vs. Ov-FBXW7+pc-NC. FBXW7, F-box and WD repeat domain containing 7; VEGF, vascular endothelial growth factor; Ov, overexpression; NC, negative control; VEGFR1/2, VEGF receptor 1/2.

Increasing evidence has suggested that angiogenesis is essential for cancer development by participating in the growth, invasion, migration and metastasis of cancer $(31,32)$. FBXW7 is a vital tumor suppressor gene, and mutations in this gene have been implicated in different types of human cancer. For example, upregulated FBXW7 expression inhibits tumor invasion, migration, EMT and angiogenesis, including oral squamous cell carcinoma, breast cancer and non-small-cell lung cancer (10,33-35). Notably, FBXW7 has been reported to act as a potent positive regulator of angiogenesis in the endothelium of the growing vasculature (15). FBXW7 expression is downregulated in OC tissues, and low FBXW7 expression is negatively associated with the malignant potential of OC (14). Increasing evidence has suggested that FBXW7-knockdown can promote OC cell invasion and migration (36). The results of the present study demonstrated that overexpression of FBXW7 inhibited the invasion, migration, EMT and angiogenesis of OC cells. To the best of our knowledge, the present study was the first to demonstrate the inhibitory effect of FBXW7 on the angiogenesis of $\mathrm{OC}$.

VEGF, a homodimeric glycoprotein, is one of the most potent and specific angiogenic factors of tumor-induced angiogenesis and binds to VEGFR1 and VEGFR2 $(18,19)$. Elevated VEGF expression has been observed in several OC cell lines and OC biopsies of different histological grades (37). A previous study has demonstrated that FBXW7 can block the effect of microRNA-182 on VEGF induction and angiogenesis in breast cancer cells (38). However, whether FBXW7 regulates VEGF expression in the progression of OC remains unclear.

The results of the present study demonstrated that overexpression of FBXW7 suppressed VEGF expression, while overexpression of VEGF partially counteracted the inhibitory effects of FBXW7 overexpression on the invasion, migration, EMT and angiogenesis of OC cells. To further elucidate the potential molecular mechanism by which FBXW7 mediates 
A

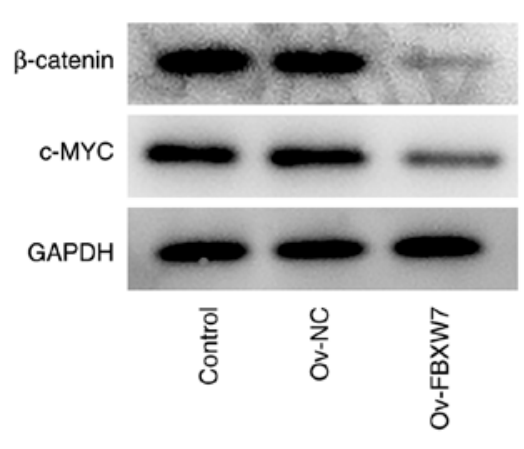

B

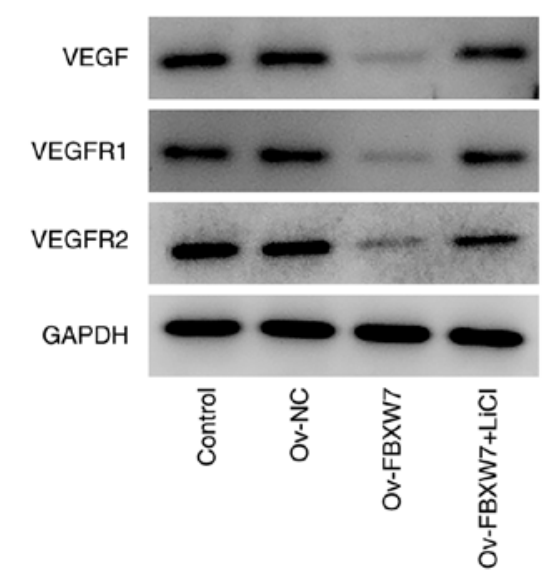

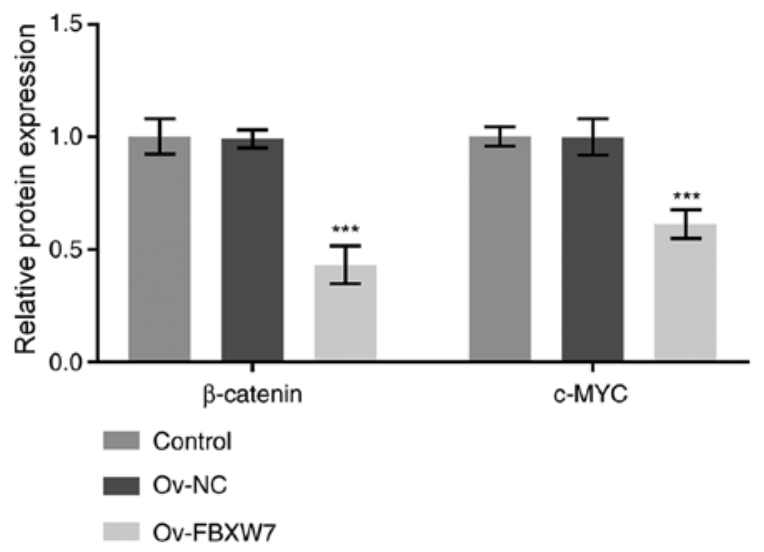

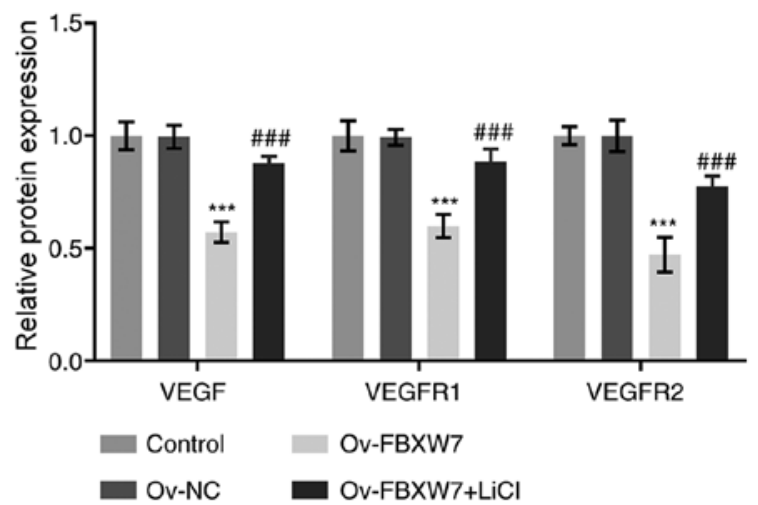

Figure 5. FBXW7 suppresses VEGF expression through inactivation of $\beta$-catenin signaling. Western blot analysis was performed to detect the protein expression levels of (A) $\beta$-catenin and c-Myc, and (B) VEGF, VEGFR1 and VEGRR2 following LiCl treatment. ${ }^{* * * *} \mathrm{P}<0.001 \mathrm{vs}$. Ov-NC; ${ }^{\# \# "} \mathrm{P}<0.001 \mathrm{vs}$. Ov-FBXW7. FBXW7, F-box and WD repeat domain containing 7; VEGF, vascular endothelial growth factor; Ov, overexpression; NC, negativecontrol; LiCl, lithium chloride.

its antitumor effects in $\mathrm{OC}$, the expression levels of key proteins in $\beta$-catenin signaling were analyzed. Increasing evidence suggests that inhibition of the $\beta$-catenin signaling pathway restrains the proliferation, invasion, migration and angiogenesis of different types of cancer, including retinoblastoma, gastric cancer and OC (39-41). Previous studies have demonstrated that $\beta$-catenin signaling helps VEGF regulate angiogenesis, and that FBXW7 promotes the degradation of $\beta$-catenin $(42,43)$. The results of the present study demonstrated that overexpression of FBXW7 inhibited the expression levels of VEGF, $\beta$-catenin and c-Myc. Notably, treatment with $\mathrm{LiCl}$, an agonist of $\beta$-catenin signaling, increased the expression levels of VEGF, VEGFR1 and VEGFR2. Overall, the current results suggested that FBXW7 may inhibit VEGF expression through inactivation of $\beta$-catenin signaling in SKOV3 cells.

In conclusion, the results of the present study demonstrated that FBXW7 inhibited the invasion, migration and angiogenesis of OC cells. To the best of our knowledge, the present study was the first to provide insight into the anti-angiogenesis effects of FBXW7 on OC, and to suggest a promising therapeutic potential of FBXW7 in the targeted treatment of this disease. However, the use of a single OC cell line to analyze FBXW7 expression and its potential mechanisms in OC is a limitation of the present study. Therefore, future studies should confirm the results of the present study using multiple OC cell lines and in vivo models.

\section{Acknowledgements}

Not applicable.

\section{Funding}

The present study was supported by the Science and Technology Planning Project of Huzhou City, Zhejiang Province (grant no. 2019GY01).

\section{Availability of data and materials}

The datasets used and/or analyzed during the current study are available from the corresponding author on reasonable request.

\section{Authors' contributions}

LZ and YP searched the literature, designed the experiments and performed the experiments. YP and JS analyzed and interpreted the data. LZ wrote the manuscript. JS revised the manuscript. LZ and JS can authenticate the raw data in the present study. All authors read and approved the final manuscript.

\section{Ethics approval and consent to participate}

Not applicable. 


\section{Patient consent for publication}

Not applicable.

\section{Competing interests}

The authors declare that they have no competing interests.

\section{References}

1. Ebell MH, Culp MB and Radke TJ: A systematic review of symptoms for the diagnosis of ovarian cancer. Am J Prev Med 50: 384-394, 2016.

2. Siegel RL, Miller KD and Jemal A: Cancer Statistics, 2017. CA Cancer J Clin 67: 7-30, 2017.

3. Reid BM, Permuth JB and Sellers TA: Epidemiology of ovarian cancer: A review. Cancer Biol Med 14: 9-32, 2017.

4. Pei H, Yang Y, Cui L, Yang J, Li X, Yang Y and Duan H: Bisdemethoxycurcumin inhibits ovarian cancer via reducing oxidative stress mediated MMPs expressions. Sci Rep 6: 28773, 2016.

5. Rodriguez-Garcia A, Sharma P, Poussin M, Boesteanu AC, Minutolo NG, Gitto SB, Omran DK, Robinson MK, Adams GP, Simpkins F, et al: CAR T Cells Targeting MISIIR for the Treatment of Ovarian Cancer and Other Gynecologic Malignancies. Mol Ther 28: 548-560, 2020.

6. Xiao G, Zhang B, Meng J, Wang J, Xu C, Tang SC, Li X, Zhang J, Liang R, Ren H, et al: miR-367 stimulates Wnt cascade activation through degrading FBXW7 in NSCLC stem cells. Cell Cycle 16: 2374-2385, 2017

7. Yeh $\mathrm{CH}$, Bellon $\mathrm{M}$ and Nicot C: FBXW7: A critical tumor suppressor of human cancers. Mol Cancer 17: 115, 2018.

8. Zhan P, Wang Y, Zhao S, Liu C, Wang Y, Wen M, Mao JH, Wei G and Zhang P: FBXW7 negatively regulates ENO1 expression and function in colorectal cancer. Lab Invest 95: 995-1004, 2015.

9. Zhang MX, Wang H and Sun GP: Tumor-suppressor Fbxw7 targets SIK2 for degradation to interfere with TORC2-AKT signaling in pancreatic cancer. Cell Biol Int 44: 1900-1910, 2020

10. Li C, Lin XF, Wang JN and Ren XS: FBXW7 inhibited cell proliferation and invasion regulated by miR-27a through PI3K/AKT signaling pathway and epithelial-to-mesenchymal transition in oral squamous cell carcinoma. Eur Rev Med Pharmacol Sci 24 3701-3709, 2020.

11. Mao JH, Perez-Losada J, Wu D, Delrosario R, Tsunematsu R, Nakayama KI, Brown K, Bryson S and Balmain A: Fbxw7/Cdc4 is a p53-dependent, haploinsufficient tumour suppressor gene. Nature 432: 775-779, 2004.

12. Zhao J, Wang Y, Mu C, Xu Y and Sang J: MAGEA1 interacts with FBXW7 and regulates ubiquitin ligase-mediated turnover of NICD1 in breast and ovarian cancer cells. Oncogene 36: 5023-5034, 2017.

13. De La Chesnaye E, Méndez JP, López-Romero R, De Los Angeles Romero-Tlalolini M, Vergara MD, Salcedo M and Ojeda SR: FBXW12, a novel F box protein-encoding gene, is deleted or methylated in some cases of epithelial ovarian cancer. Int J Clin Exp Pathol 8: 10192-10203, 2015.

14. Kitade S, Onoyama I, Kobayashi H, Yagi H, Yoshida S, Kato M Tsunematsu R, Asanoma K, Sonoda K, Wake N, et al: FBXW7 is involved in the acquisition of the malignant phenotype in epithelial ovarian tumors. Cancer Sci 107: 1399-1405, 2016.

15. Izumi N, Helker C, Ehling M, Behrens A, Herzog W and Adams RH: Fbxw7 controls angiogenesis by regulating endothelial Notch activity. PLoS One 7: e41116, 2012.

16. Rivera LB and Bergers G: CANCER. Tumor angiogenesis, from foe to friend. Science 349: 694-695, 2015.

17. Ye W, Ni Z, Yicheng S, Pan H, Huang Y, Xiong Y and Liu T: Anisomycin inhibits angiogenesis in ovarian cancer by attenuating the molecular sponge effect of the lncRNA Meg3/miR 421/PDGFRA axis. Int J Oncol 55: 1296-1312, 2019.

18. Carmeliet P: VEGF as a key mediator of angiogenesis in cancer. Oncology 69 (Suppl 3): 4-10, 2005.

19. Sadremomtaz A, Mansouri K, Alemzadeh G, Safa M, Rastaghi AE and Asghari SM: Dual blockade of VEGFR1 and VEGFR 2 by a novel peptide abrogates VEGF-driven angiogenesis, tumor growth, and metastasis through PI3K/AKT and MAPK/ERK1/2 pathway. Biochim Biophys Acta Gen Subj 1862: 2688-2700, 2018.

20. Livak KJ and Schmittgen TD: Analysis of relative gene expression data using real-time quantitative PCR and the 2(-Delta Delta C(T)) Method. Methods 25: 402-408, 2001.
21. Ai B, Bie Z,Zhang S and Li A: Paclitaxel targets VEGF-mediated angiogenesis in ovarian cancer treatment. Am J Cancer Res 6: 1624-1635, 2016.

22. Xu J,Zhang P, Sun H and Liu Y: LINC01094/miR-577 axis regulates the progression of ovarian cancer. J Ovarian Res 13: 122, 2020.

23. Kappes L, Amer RL, Sommerlatte S, Bashir G, Plattfaut C, Gieseler F, Gemoll T, Busch H, Altahrawi A, Al-Sbiei A, et al: Ambrisentan, an endothelin receptor type A-selective antagonist, inhibits cancer cell migration, invasion, and metastasis. Sci Rep 10: 15931, 2020.

24. Ombrato L and Malanchi I: The EMT universe: Space between cancer cell dissemination and metastasis initiation. Crit Rev Oncog 19: 349-361, 2014.

25. Ji L, Li X, Zhou Z, Zheng Z, Jin L and Jiang F: LINC01413/ hnRNP-K/ZEB1 axis accelerates cell proliferation and EMT in colorectal cancer via inducing YAP1/TAZ1 translocation. Mol Ther Nucleic Acids 19: 546-561, 2020.

26. Peng Y, Li Y, Li Y, Wu A, Fan L, Huang W, Fu C, Deng Z, Wang K, Zhang Y, et al: HOXC10 promotes tumour metastasis by regulating the EMT-related gene Slug in ovarian cancer. Aging (Albany NY) 12: 19375-19398, 2020.

27. Thiery JP: Epithelial-mesenchymal transitions in tumour progression. Nat Rev Cancer 2: 442-454, 2002.

28. Xin L, Zhao R, Lei J, Song J, Yu L, Gao R, Ha C, Ren Y, Liu X, Liu Y, et al: SND1 acts upstream of SLUG to regulate the epithelial-mesenchymal transition (EMT) in SKOV3 cells. FASEB J 33: 3795-3806, 2019.

29. Folkman J: Angiogenesis. Annu Rev Med 57: 1-18, 2006.

30. Folkman J: Tumor angiogenesis: Therapeutic implications. N Engl J Med 285: 1182-1186, 1971.

31. Zheng Y, Chen H, Zhao Y, Zhang X, Liu J, Pan Y, Bai J and Zhang H: Knockdown of FBXO22 inhibits melanoma cell migration, invasion and angiogenesis via the HIF-1 $\alpha /$ VEGF pathway. Invest New Drugs 38: 20-28, 2020.

32. Chen L, Lin G, Chen K, Liang R, Wan F, Zhang C, Tian G and Zhu X: VEGF promotes migration and invasion by regulating EMT and MMPs in nasopharyngeal carcinoma. J Cancer 11: 7291-7301, 2020.

33. Wu XP, Chen H, Wu MT, Peng SG and Zhang L: Downregulation of miR-182-5p inhibits the proliferation and invasion of triple-negative breast cancer cells through regulating TLR4/NF-kappa B pathway activity by targeting FBXW7. Ann Transl Med 8: 13,2020.

34. Chiang $\mathrm{CH}$, Chu PY, Hou MF and Hung WC: MiR-182 promotes proliferation and invasion and elevates the HIF-1 alpha-VEGF-A axis in breast cancer cells by targeting FBXW7. Am J Cancer Res 6: 1785-1798, 2016.

35. Xiao G, Li Y, Wang M, Li X, Qin S, Sun X, Liang R, Zhang B, Du N, Xu C, et al: FBXW7 suppresses epithelial-mesenchymal transition and chemo-resistance of non-small-cell lung cancer cells by targeting snail for ubiquitin-dependent degradation. Cell Prolif 51: e12473, 2018.

36. Guo Y, Zhang Z, Wang Z, Liu G, Liu Y and Wang H: Astragalus polysaccharides inhibit ovarian cancer cell growth via microRNA-27a/FBXW7 signaling pathway. Biosci Rep: Mar 17, 2020 (Epub ahead of print). doi: 10.1042/BSR20193396.

37. Inan S, Vatansever S, Celik-Ozenci C, Sanci M, Dicle N and Demir R: Immunolocalizations of VEGF, its receptors flt-1, KDR and TGF-beta's in epithelial ovarian tumors. Histol Histopathol 21: 1055-1064, 2006.

38. Chiang CH, Chu PY,Hou MF and Hung WC: MiR-182 promotes proliferation and invasion and elevates the HIF-1 $\alpha$-VEGF-A axis in breast cancer cells by targeting FBXW7. Am J Cancer Res 6: 1785-1798, 2016

39. Liao YJ, Yin XL, Deng Y and Peng XW: PRC1 gene silencing inhibits proliferation, invasion, and angiogenesis of retinoblastoma cells through the inhibition of the Wnt $/ \beta$-catenin signaling pathway. J Cell Biochem 120: 16840-16852, 2019.

40. Wang F, Zhu W, Yang R, Xie W and Wang D: LncRNA ZEB2-AS1 contributes to the tumorigenesis of gastric cancer via activating the Wnt//-catenin pathway. Mol Cell Biochem 456: 73-83, 2019.

41. Dai J, Wei R, Zhang P and Kong B: Overexpression of microRNA-195-5p reduces cisplatin resistance and angiogenesis in ovarian cancer by inhibiting the PSAT1-dependent GSK3 $\beta$ / $\beta$-catenin signaling pathway. J Transl Med 17: 190, 2019.

42. Shi L, Yang F, Luo F, Liu Y, Zhang F, Zou M and Liu Q: Evodiamine exerts anti-tumor effects against hepatocellular carcinoma through inhibiting $\beta$-catenin-mediated angiogenesis. Tumour Biol 37: 12791-12803, 2016.

43. Jiang JX, Sun CY, Tian S, Yu C, Chen MY and Zhang H: Tumor suppressor Fbxw7 antagonizes WNT signaling by targeting $\beta$-catenin for degradation in pancreatic cancer. Tumour Biol 37: 13893-13902, 2016.

This work is licensed under a Creative Commons Attribution-NonCommercial-NoDerivatives 4.0 International (CC BY-NC-ND 4.0) License. 